\title{
YTHDF1 promotes breast cancer progression by facilitating FOXM1 translation in an m6A-dependent manner
}

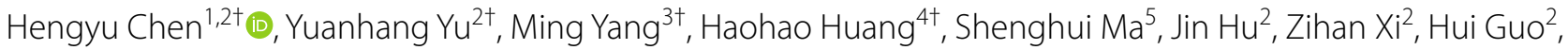
Guojie Yao ${ }^{4}$, Liu Yang ${ }^{4}$, Xiaoqing Huang ${ }^{2}$, Feng Zhang ${ }^{6}$, Guanghong Tan ${ }^{1 *}$, Huangfu Wu ${ }^{1 *}$, Wuping Zheng ${ }^{1 *}$ and Lei Li $i^{2^{*}}$

\begin{abstract}
Background: $\mathrm{N} 6$-methyladenosine $\left(\mathrm{m}^{6} \mathrm{~A}\right)$ is the most common post-transcriptional modification at the RNA level. However, the exact molecular mechanisms of $\mathrm{m} 6 \mathrm{~A}$ epigenetic regulation in breast cancer remain largely unknown and need to be fully elucidated.

Methods: The integrating bioinformatics analyses were used to screen clinical relevance and dysregulated m6A "reader" protein YTHDF1 in breast cancer from TCGA databases, which was further validated in a cohort of clinical specimens. Furthermore, functional experiments such as the CCK-8 assay, EdU assay, wound healing assay, transwell invasion assay and cell cycle assay were used to determine the biological role of YTHDF1 in breast cancer. RIP, m6A-IP, and CLIP assays were used to find the target of YTHDF1 and further verification by RT-qPCR, western blot, polysome profiling assay. The protein-protein interaction between YTHDF1 and FOXM1 was detected via co-immunoprecipitation.
\end{abstract}

Results: Our study showed that YTHDF1 was overexpressed in breast cancer cells and clinical tissues specimens. At the same time, the high expression level of YTHDF1 was positively correlated with tumor size, lymph node invasion, and distant metastasis in breast cancer patients. YTHDF1 depletion repressed the proliferation, invasion and epithelialmesenchymal transformation (EMT) and induced G0/G1 phase cell cycle arrest of breast cancer cells in vitro and in vivo. We also demonstrated that FOXM1 is a target of YTHDF1. Through recognizing and binding to the m6A-modified mRNA of FOXM1, YTHDF1 accelerated the translation process of FOXM1 and promoted breast cancer metastasis. Whereas overexpression of FOXM1 in breast cancer cells partially counteracted the tumor suppressed effects caused by YTHDF1 silence, which further verified the regulatory relationship between YTHDF1 and FOXM1.

\footnotetext{
*Correspondence: tanhoho@163.com; wuhuangfu1103@163.com; hnzwp2000@163.com; leili2008@hust.edu.cn

${ }^{\dagger}$ Hengyu Chen, Yuanhang Yu, Ming Yang and Haohao Huang contributed equally and are co-first authors

${ }^{1}$ Department of Breast and Thyroid Surgery, The Second Affiliated

Hospital of Hainan Medical University, Key Laboratory of Tropical

Translational Medicine of Ministry of Education \& Hainan Provincial Key

Laboratory of Tropical Medicine, Haikou 570311, China

${ }^{2}$ Department of Breast and Thyroid Surgery, Union Hospital, Tongji

Medical College, Huazhong University of Science and Technology, Wuhan 430022, China

Full list of author information is available at the end of the article
} permits use, sharing, adaptation, distribution and reproduction in any medium or format, as long as you give appropriate credit to the original author(s) and the source, provide a link to the Creative Commons licence, and indicate if changes were made. The images or other third party material in this article are included in the article's Creative Commons licence, unless indicated otherwise in a credit line to the material. If material is not included in the article's Creative Commons licence and your intended use is not permitted by statutory regulation or exceeds the permitted use, you will need to obtain permission directly from the copyright holder. To view a copy of this licence, visit http://creativecommons.org/licenses/by/4.0/. The Creative Commons Public Domain Dedication waiver (http://creativeco mmons.org/publicdomain/zero/1.0/) applies to the data made available in this article, unless otherwise stated in a credit line to the data. 
Conclusion: Our study reveals a novel YTHDF1/FOXM1 regulatory pathway that contributes to metastasis and progression of breast cancer, suggesting that YTHDF1 might be applied as a potential biomarker and therapeutic target. That also advances our understanding of the tumorigenesis for breast cancer from $\mathrm{m} 6 \mathrm{~A}$ epigenetic regulation.

Keywords: Breast cancer, YTHDF1, FOXM1, m6A, EMT

\section{Background}

$\mathrm{m}^{6} \mathrm{~A}$ modification is the most abundant RNA modification in eukaryotic cells [1], and mainly targets messenger RNA (mRNA) and non-coding RNA (ncRNA). $\mathrm{m}^{6} \mathrm{~A}$ modification is reversible, and the methylation and demethylation processes were performed by specific methyltransferases (writers) and demethylases (erasers), respectively [2]. $\mathrm{m}^{6} \mathrm{~A}$ modification does not change the sequence of RNA base. It regulates gene expression by affecting the structure of RNA to alter its interaction with $\mathrm{m}^{6} \mathrm{~A}$ binding protein (readers) or other protein complexes. $\mathrm{m}^{6} \mathrm{~A}$ modification occurs in more than $30 \%$ of mRNAs in mammals, with an average of $3-5 \mathrm{~m}^{6} \mathrm{~A}$ modifications per mRNA [3]. $\mathrm{m}^{6} \mathrm{~A}$ modification is essential for regulating gene expression at multiple levels (transcription, post-transcription, translation and post-translation) and influencing critical physiological and pathological processes [3]. The physiological processes affected by $\mathrm{m}^{6} \mathrm{~A}$ modification mainly include DNA repair, differentiation of hematopoietic stem cells, the development of tissues and embryos, etc. In addition, the abnormal expression of the writers, erasers and readers is also an important cause of tumorigenesis [4-8].

YTH N6-Methyladenosine RNA Binding Protein 1 (YTHDF1) is a member of the YT521-B homologous (YTH) domain protein family $[9,10]$. It is highly conserved in evolution, and it regulates gene expression by binding to $\mathrm{m}^{6} \mathrm{~A}$-modified mRNAs through its $\mathrm{C}$-terminal YTH domain, thereby changing the fate of genes $[10,11]$. Although the YTH family members have similar functional domains, they have different regulatory effects on the target mRNA. The role of YTHDF2, the first reader to be identified, is to guide the mRNAs to the decay sites (such as the processing body) to induce their degradation $[12,13]$. YTHDF1, on the other hand, promotes the translation of $\mathrm{m}^{6} \mathrm{~A}$-modified mRNAs by interacting with transcription initiation factors $[14,15]$. When the YTH proteins are abnormally expressed, the degradation and translation of oncogenes or tumor suppressor genes are disorganized, and tumorigenesis is triggered [16].

Recent studies have shown that YTHDF1 is highly expressed and associated with poor prognosis in various tumor tissues such as colon cancer [17], melanoma [18], liver cancer [19], and non-small cell lung cancer [20]. In breast cancer, although it has been reported that YTHDF1 expression is significantly increased in tumor tissues compared with para-cancerous tissues and is closely associated with poor prognosis [21], its specific mechanisms have not yet been clarified.

In the present study, we found that YTHDF1 was aberrantly expressed in breast cancer tissues by analyzing the cancer genome atlas (TCGA) database and further verified this finding via breast patient samples and cell lines. Moreover, patients with high YTHDF1 expression had poor overall survival (OS), and this survival difference was more distinct in the HER2+ subgroup. Data from our clinical specimens indicated that YTHDF1 was also positively correlated with tumor size, lymph node invasion as well as distant metastasis in breast cancer patients. Subsequently, a series of functional experiments confirmed that YTHDF1 promoted the proliferation and invasion of breast cancer cells in vitro and in vivo. In brief, we demonstrated that YTHDF1 binds to the $\mathrm{m}^{6} \mathrm{~A}$-modified FOXM1 gene at the coding sequence (CDS) region and plays its role as an oncogenic gene by promoting FOXM1 translation rather than transcription, exacerbating the progression of breast cancer.

\section{Materials and methods \\ Patient samples and cell lines}

Breast cancer and para-cancerous tissues of 48 patients were collected from the Department of Breast and Thyroid Surgery, Union Hospital, Tongji Medical College, Huazhong University of Science and Technology from June 2019 to December 2020. After careful examination of the tissues by three pathologists, the patients were diagnosed with breast cancer. Patients who received any treatment (including surgery, chemotherapy, immunotherapy, etc.) before the surgery were excluded from the study. Informed consent was signed by all patients. The study was approved by the Ethics Committee of Tongji Medical College, Huazhong University of Science and Technology. The study was in accordance with the Declaration of Helsinki.

The cell lines used in the study included human normal breast cell line MCF-10A, human breast cancer cell line MCF-7, MDA-MB-231, SKBR3, T47D and BT-549. MCF10A, MCF-7, MD-MB-231, T47D and BT-549 were purchased from American Type Culture Collection (ATCC). SKBR3 was purchased from China Center for Type Culture Collection (CCTCC). Cells except for MCF-10A were cultured in DMEM medium supplemented with 
10\% FBS (Gibco, US) and antibiotics (Penicillin $100 \mathrm{U} / \mathrm{ml}$, Streptomycin $100 \mathrm{mg} / \mathrm{ml}$ ) (Gibco, US). For MCF-10A, the culture conditions were: mixture of DMEM and F12 medium (1:1) (Gibco, US), antibiotics (Pen/Strep), 5\% horse serum (Ausbian, AUS), insulin $(10 \mu \mathrm{g} / \mathrm{ml})$ (Thermo Fisher Scientific, US), epidermal growth factor $(20 \mathrm{ng} / \mathrm{ml})$ (Gibco, US), choleramycin (100 ng/ml) (Sigma, US) and hydrocortisone $(0.5 \mu \mathrm{g} / \mathrm{ml})$ (Tocris, US). Cells were cultured at $37{ }^{\circ} \mathrm{C}$ with $5 \% \mathrm{CO}_{2}$. Cells were cultured for up to 20 passages.

\section{Cell proliferation assay}

Cell proliferation was measured by CCK-8 (Cell Counting Kit-8, Dojindo, Japan) and EdU assays. For the CCK-8 assay, $1 * 10^{3}$ cells were seeded and cultured in 96-well plates for $24 \mathrm{~h}, 48 \mathrm{~h}, 72 \mathrm{~h}, 96 \mathrm{~h}$ and $120 \mathrm{~h} ; 10 \mu \mathrm{l}$ CCK- 8 solution was added to each well. After adding the solution, the culture was continued for $4 \mathrm{~h}$, and the absorbance was measured every $30 \mathrm{~min}$ using a microplate reader at $450 \mathrm{~nm}$.

EdU proliferation assay was carried out using ClickiT Plus EdU Alexa Fluor 594 flow cytometry assay kit (Thermo Fisher Scientific, Catalog No. C10646) according to the manufacturer's instructions. Images were captured by the confocal microscope (Olympus, Japan) at ten randomized fields.

\section{Cell cycle assay}

$2 * 10^{5}$ cells were seeded in a 12 -well plate for $48 \mathrm{~h}$, and then cell cycle assays were performed. The procedures are briefly described: cells were collected by centrifugation at $8500 \mathrm{rpm}$ for $5 \mathrm{~min}$, and $70 \%$ cold ethanol was added to the cells dropwise. Cells were then fixed at $4{ }^{\circ} \mathrm{C}$ for $30 \mathrm{~min}$. Next, wash the cells with $1 \times$ Phosphate Buffer Saline (PBS) twice. After staining the cells with propidium iodide (PI) antibody for $30 \mathrm{~min}$, the cell cycle was measured by flow cytometry.

\section{Cell invasion assay}

Breast cancer cell invasive ability was measured by transwell insert chambers (Corning, NY, USA) pre-coated with Matrigel (Corning Inc.). In brief, $2 * 10^{4}$ cells were seeded into the upper chamber containing $200 \mu \mathrm{l}$ serumfree medium, and $500 \mu \mathrm{l}$ culture medium with $20 \%$ FBS was added into the bottom chamber. After $24 \mathrm{~h}$, invading cells were fixed with $4 \%$ paraformal-dehyde and stained with $0.1 \%$ crystal violet. Cells were counted using a microscope for 5 fields randomly at $100 \times$ magnification.

\section{Immunohistochemistry and HE staining}

Hematoxylin and eosin (HE) staining was conducted following the standard protocol. For immunohistochemistry staining, tissue slides $(6 \mu \mathrm{m})$ were deparaffinized and hydrated. Antigen retrieval was performed using citrate solution (PH 6), and the tissues were then blocked with $2 \%$ BSA for $2 \mathrm{~h}$ at room temperature. Next, tissues were incubated overnight with primary antibody (antiYTHDF1: Abcam, catalog No. 230330) at $4{ }^{\circ} \mathrm{C}$. Subsequently, tissues were incubated with HRP-conjugated antibody for at least $1 \mathrm{~h}$ in the darkroom at room temperature. Images were captured with the microscope (Leitz, Italy), and the results were analyzed using ImageJ software (version 1.8.0, NIH, US). The IRS (immunoreactive score) scoring system to determine immunohistochemical positivity and the protein expression levels. $I R S=S I$ (Staining Index) * PP (Proportion of Positive tumor cells). SI ranges from 0 to 3 and PP from 0 to 4 . IRS score ranges from 0 to 12 . For protein expression, IRS $0-1$ : no expression, 2-3: low expression, 4-8: moderate expression, 9-12: high expression [22]. The mean integrated optical density (IOD) values of YTHDF1 and FOXM1 in tumor tissues were calculated by Image Pro-Plus 6.0 software (MEDIA CYBERNETICS, US).

\section{RNA immunoprecipitation (RIP)}

RIP was carried out to detect mRNAs that bind to the YTHDF1 protein. The RIP was conducted using a Megna RNA-binding protein kit (Millipore, US). $10 \mu \mathrm{l}$ YTHDF1 or IgG antibody (Cell signaling Technology, US) was incubated with protein $\mathrm{A} / \mathrm{G}$ magnetic beads for at least $4 \mathrm{~h}$ at $4{ }^{\circ} \mathrm{C}$. After lysing the cells with RIP buffer, the cells were incubated with the washed magnetic beads at $4{ }^{\circ} \mathrm{C}$ overnight. Then RNA was extracted by Trizol reagent (Invitrogen, US), and the expression of the target genes was detected by RT-qPCR.

\section{Cross-linking immunoprecipitation (CLIP)}

The CLIP assay was conducted according to the protocol as previously described [23]. Briefly, $2 * 10^{7}$ MCF-7 cells were irradiated with the UV crosslinker (Thermo Fisher Scientific, US) (wavelength $260 \mathrm{~nm}$ ). The cells were then lysed with the RIPA buffer and co-incubated with protein $\mathrm{A} / \mathrm{G}$ magnetic beads at $4{ }^{\circ} \mathrm{C}$ for at least $4 \mathrm{~h}$. The immune complexes were then eluted at $60{ }^{\circ} \mathrm{C}$ for 20 min with a $50 \mathrm{mM}$ Tris $-\mathrm{HCl}$ solution ( $\mathrm{pH}$ 7.8). To isolate the RNA from the immune complexes, we use chloroform to extract RNA. Finally, RT-qPCR was used to detect and analyze the expression level of the target genes.

\section{$m^{6} \mathrm{~A}$ immunoprecipitation ( $\left.\mathrm{m}^{6} \mathrm{~A}-\mathrm{IP}\right)$}

$1.5 \mu \mathrm{g}$ of IgG or $\mathrm{m}^{6} \mathrm{~A}$ antibody was conjugated to protein A/G beads at $4{ }^{\circ} \mathrm{C}$ overnight. Next, incubate $300 \mu \mathrm{g}$ fragmented RNA with the IgG or $\mathrm{m}^{6} \mathrm{~A}$ antibody in the immunoprecipitation buffer containing RNase inhibitor at $4{ }^{\circ} \mathrm{C}$ overnight. RNA was eluted from the beads using 
elution buffer and was extracted for RT-qPCR by phenol and ethanol.

\section{Polysome profiling}

Polysome profiling was conducted according to the protocols described previously [24]. After treated with $0.1 \mathrm{mg} / \mathrm{ml}$ cycloheximide for $10 \mathrm{~min}$, cells were washed with cold PBS and lysed with polysome lysis buffer (10 mM Tris-HCl, pH 7.4; $10 \mathrm{mM} \mathrm{MgCl}_{2} ; 0.3 \mathrm{mM}$ $\mathrm{NaCl} ; 1 \%$ Triton X-100; RNase inhibitor and $100 \mu \mathrm{g} / \mathrm{ml}$ cycloheximide). Cell lysates were centrifuged at $2000 \mathrm{rpm}$ for $5 \mathrm{~min}$ and 12,000 rpm for $15 \mathrm{~min}$. RNA concentration was detected by Nanodrop (Thermo Fisher Scientific, US). $500 \mu \mathrm{g}$ RNA was then added in freshly prepared sucrose gradient solutions $(10-50 \%)$ and centrifuged at $30,000 \mathrm{rpm}$ for $4 \mathrm{~h}$ at $4{ }^{\circ} \mathrm{C} .1 \mathrm{ml}$ fractions were collected. Total RNA was extracted by RNeasy Mini Kit (QIAGEN, US) for qRT-PCR analysis.

\section{Lentivirus production and cell transduction}

HEK-293 T cells were used to produce lentivirus particles. Briefly, pLKO.1 puro-sh-YTHDF1\#1/sh-YTHDF1\#2 or pLKO.1 puro control vector, helper vector pxPAX2 and envelope vector pMD2.G were transfected into HEK$293 \mathrm{~T}$ cells using Lipofectamine 2000 (Thermo Fisher Scientific, US). Supernatants containing lentivirus particles were collected and filtered after $48 \mathrm{~h}$ transfection. $1 * 10^{4}$ cells were incubated with $1 \mathrm{ml}$ supernatants for $24 \mathrm{~h}$ and were selected using $4 \mu \mathrm{g} / \mathrm{ml}$ puromycin. $1 \mu \mathrm{g} / \mu \mathrm{l}$ puromycin was used as a selective pressure to get stably transfected cell lines. sh-YTHDF1\#1 and sh-YTHDF1\#2 were synthesized by GenePharma $(\mathrm{CN})$. Oligo sequence of sh-YTHDF1\#1: 5'-CGGTGGGACAAATGTGAACAT3'; sh-YTHDF1\#2: 5'-CCCGAAAGAGTTTGAGTG GAA-3'; sh-YTHDF1-3: 5'-GTTCGTTACATCAGAAGG ATA-3'. sh-FOXM1\#1: 5'-GCTGGGATCAAGATTATT A-3'; sh-FOXM1\#2: 5'-GGCTGCACTATCAACAAT A-3'. Scramble shRNA was purchased from Addgene (\#1864, US).

\section{Cell transfection}

Transfection of plasmids was performed using Lipofectamine 2000 reagent (Invitrogen) according to the manufacturer's instruction. YTHDF1-WT (Addgene \#70087), YTHDF1-MUT, FOXM1-WT (Addgene \#68811, FOXM1 isoform 3) and FOXM1-MUT, CCNB1 (Addgene \#39871) overexpression plasmids were used for transfection. Detailed information on the mutations was shown in Additional file 2: Fig. S2. The sequences of siRNAs targeting YTHDF1 (si-YTHDF1) were as follows: siYTHDF1-1: 5'-GAACAAAAGGACAAGAUAAUA-3', si-YTHDF1-2: 5'-CAAAAGGACAAGAUAAUAAAG-3'.

\section{RT-qPCR}

Total RNA was extracted from cells using TRIzol Reagent (Life Technologies) according to the manufacturer's instructions. RNA was transcribed into cDNA using the Reverse Transcription Kit (Takara, JP). RT-qPCR was conducted by SYBR Green Master Mix (\#4309155, Applied Biosystems, US). The procedure was set as follows: Hold $95{ }^{\circ} \mathrm{C}$ for $10 \mathrm{~min}$; Denature $95{ }^{\circ} \mathrm{C} 15 \mathrm{~s}$ and Anneal/Extend $60{ }^{\circ} \mathrm{C} 1 \mathrm{~min}$ for 40 cycles. Primers were listed in Additional file 5: Table S1.

\section{Western blot and antibodies}

Cells were washed with cold Phosphate Buffered Saline (PBS, Thermo Fisher Scientific, US) twice before protein extraction. Total proteins were extracted from cells using RIPA buffer (Sigma Aldrich, US) supplemented with the protease inhibitor cocktail (Roche, Shanghai, CN) according to the manufacturer's instructions. 25-30 $\mu \mathrm{g}$ proteins were loaded onto the 4-12\% SDS-PAGE (BeyoGel, CN). Electrophoresis was performed at $120 \mathrm{~V}$ for $1 \mathrm{~h}$. Proteins were then transferred to the PVDF membranes at $250 \mathrm{mAh}$ for $2 \mathrm{~h}$. After blocking with $5 \%$ non-fat milk for $30 \mathrm{~min}$, membranes were incubated with primary antibodies overnight at $4{ }^{\circ} \mathrm{C}$. Western blot images were captured by a myECL imager (Thermo Fisher Scientific, US). Primary antibodies used in the present study were listed as follows: FOXM1 (A2493, ABclonal), YTHDF1 (17479-1-AP, Proteintech), ZEB1 (21544-1-AP, Proteintech), N-cadherin (22018-1-AP, Proteintech), E-cadherin (20874-1-AP, Proteintech), Vimentin (10366-1-AP, Proteintech), Snail (ab216347, Abcam), $\beta$-Actin (ab8226, Abcam), anti-Flag tag (SAB4301135, Sigma-Aldrich), anti-HA-tag (05-904, Sigma-Aldrich).

\section{In vivo assays}

Animal Experiments were conducted under the approval of the ethics committee of Tongji Medical College, Huazhong University of Science and Technology (Protocol \#TJU 2018-0025). NOD/SCID immune-deficient mice were purchased from Shanghai Experimental Animal Center. $2 * 10^{6}$ MCF-7 cells transduced with sh-NC or sh-YTHDF1-were subcutaneously injected into the mice (5/group). Tumor width and length were measured every 7 days. Tumor volume $=\left(\right.$ length ${ }^{*}$ width $\left.^{2}\right) / 2$. After 7 weeks, mice were sacrificed, and the weight of tumors was detected. Xenografts were collected for HE staining, immunohistochemistry staining and western blot analysis.

For spontaneous lung metastasis assay, $4 * 10^{6}$ sh-NC or sh-YTHDF1\#2 transduced MCF-7 cells were injected into the mammary fat pads of the NOD/SCID mice (5/group). The primary tumor was removed when 
its volume reached $150 \mathrm{~mm}^{3}$. The mice were sacrificed, and lung metastasis nodules were counted 12 weeks after the removal.

\section{Statistical analysis}

Data were expressed as mean \pm standard derivation (SD). A T-test was used for comparison between two groups. A one-way ANOVA test followed by an SNK test was used for comparison between three groups. Kaplan-Meier curve was used for survival analysis. All data were analyzed using GraphPad Prism 9.0 (GraphPad Software, US). Statistical significance is considered as ${ }^{*} \mathrm{P}<0.05,{ }^{* *} \mathrm{P}<0.01,{ }^{* * *} \mathrm{P}<0.001$. All experiments were three independent repetitions.

\section{Results}

\section{YTHDF1 is highly expressed in breast cancer samples} and cells

To identify the protein related to $\mathrm{m} 6 \mathrm{~A}$ modification that potentially drives the tumorigenesis of breast cancer, we first explored the expression of m6A "reader' protein in the TCGA-BRCA dataset. The result showed that the expression of YTHDF1 in breast cancer samples was significantly higher than that in the normal control group, but there was no significant difference in the expression of YTHDF1 in breast cancer patients of different stages and subtypes (Fig. 1A, Additional file 1: Fig. S1A, B). Similar results were obtained in the collected breast cancer tissues and adjacent normal tissues validated by RT-qPCR, western blot analysis and immunohistochemical staining (Fig. 1B, G, H). We further explored

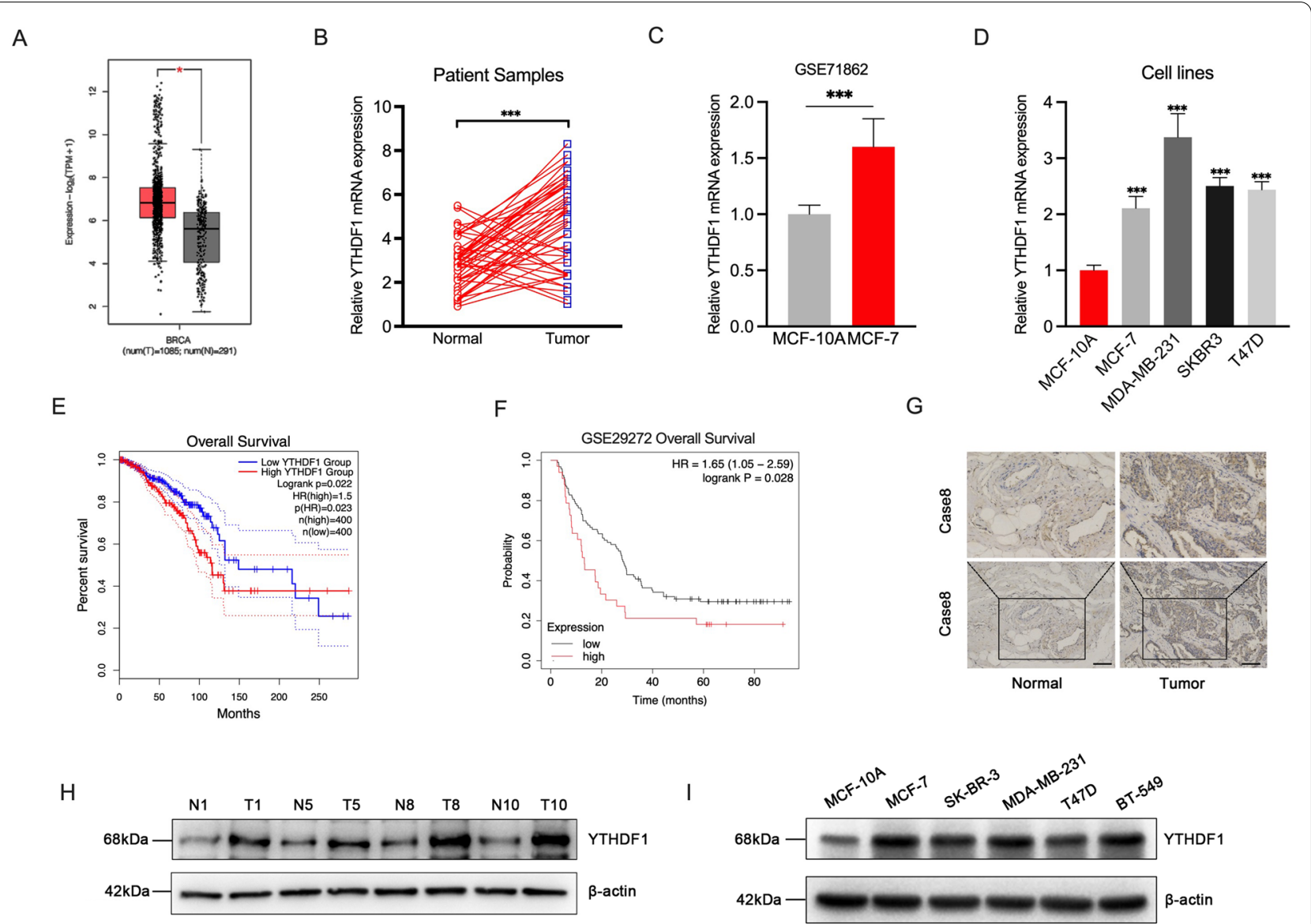

Fig. 1 YTHDF1 was highly expressed in breast cancer samples and cells. A YTHDF1 expression in breast cancer tissues and normal tissues from TCGA-BRCA dataset. Relative YTHDF1 mRNA expression in B collected breast cancer tissues and adjacent normal tissues samples, C MCF-10A and MCF-7 cells from GSE71862 dataset and D normal human breast cell line MCF-10A and breast cancer cell line MCF-7, MDA-MB-231, SKBR3, T47D and BT-549. Kaplan-Meier curves of OS in the TCGA dataset $\mathbf{E}$ and GSE29272 dataset $\mathbf{F}$ of all breast cancer patients with high and low YTHDF1 expression. G Immunohistochemical staining of YTHDF1 in a breast cancer sample. YTHDF1 protein expression in $\mathbf{H}$ breast cancer tissues and adjacent normal tissues and I normal human breast cell line MCF-10A and breast cancer cell line MCF-7, MDA-MB-231, SKBR3, T47D and BT-549. All data were presented as means $\pm S D$ of at least three independent repetitions. Values are significant at ${ }^{*} P<0.05,{ }^{* *} P<0.01,{ }^{* * *} P<0.001$ as indicated 
the expression of YTHDF1 in cell lines. The results of the GSE71862 dataset showed that the expression of YTHDF1 in breast cancer cell line MCF-7 was 1.6 times that of human normal breast cell line MCF-10A (Fig. 1C). RT-qPCR and western blot analysis in the cell lines also suggested that the mRNA and protein expressions of YTHDF1 were significantly increased in the breast cancer cell lines (Fig. 1D, I). We also observed the effects of YTHDF1 on the prognosis of breast cancer patients. Kaplan-Meier analysis of TCGA-BRCA and GSE29272 datasets indicated that increased YTHDF1 was associated with shortened OS, which was a marker of poor prognosis in breast cancer (Fig. 1E, F). In addition, by stratification of TCGA-BRCA data, we also found that increased YTHDF1 could further distinguish the prognosis of the patients in the HER2+ and Luminal subtypes. Its high expression was significantly associated with poor prognosis (Additional file 1: Fig. S1C-E). To verify the clinical significance of YTHDF1 for breast cancer, we collected and examined its expression as well as the clinicopathologic features in a cohort of patients. The result indicated that the high expression level of YTHDF1 was positively correlated with tumor size, lymph node invasion, and distant metastasis in breast cancer patients (Additional file 5: Table S2). Taken together, these results demonstrate that YTHDF1 was dysregulated in breast cancer and that high expression of YTHDF1 was associated with poor outcomes in patients with breast cancer.

\section{YTHDF1 knockdown inhibits the growth ability and arrests the cell cycle of breast cancer cells}

We transduced shRNAs targeting YTHDF1 into breast cancer cell lines MDA-MB-231 and MCF-7 to knock down the expression of YTHDF1. After $48 \mathrm{~h}$ transduction, we examined the mRNA expression of YTHDF1 to verify the knockdown efficiency, and the results showed that all three shRNAs significantly suppressed the expression of YTHDF1 in MDA-MB-231 and MCF-7 cells (Fig. 2A). We then performed CCK-8 and EdU cell proliferation assays using scramble shRNA transduced MDAMB-231 and MCF-7 cells (sh-NC) and sh-YTHDF1 transduced cancer cells. CCK- 8 results indicated that the proliferation of cells in the sh-YTHDF1 group was significantly inhibited after $48 \mathrm{~h}$ incubation compared to the cells in the sh-NC group (Fig. 2B, C). EdU cell proliferation assay also showed that after $2 \mathrm{~h}$ incubation with the EdU medium, the proliferation of breast cancer cells in the sh-YTHDF1 group was significantly lower than that in the sh-NC group (Fig. 2D). In addition, we also investigated the effects of YTHDF1 on the cell cycle, and the results suggested that G0/G1 cell cycle arrest occurred in the sh-YTHDF1 group. (Fig. 2E).

\section{Silence of YTHDF1 restrains cell migration, invasion ability} and EMT

We then investigated the role of YTHDF1 on the invasion capability of breast cancer cells. Transwell assay and wound healing assay revealed that knockdown of YTHDF1 could effectively suppress the migration and invasion ability of MDA-MB-231 and MCF-7 cells (Fig. 3A, B). Epithelial-mesenchymal transformation (EMT) was reported to be the first step of metastasis and played an essential role in breast cancer progression. Several studies have reported that YTHDF1 is involved in the molecular regulation pathways of EMT. Therefore, EMT-related proteins were examined by immunoblot assay, and the results showed that compared with the control group, the mesenchymal protein expressions of N-cadherin, Vimentin, ZEB1 and Snail in the sh-YTHDF1 group were significantly decreased. In contrast, epithelial marker protein E-cadherin was upregulated (Fig. 3C).

To ensure the accuracy of the experiment, similar experiments were carried out using si-YTHDF1 transfected MCF-7 cells. The results showed that after YTHDF1 knockdown with siYTHDF1, the proliferative and invasive ability of MCF-7 cells were significantly reduced, and the EMT was also inhibited (Additional file 3: Fig. S3).

\section{Depletion of YTHDF1 inhibits the growth of breast cancer cells in vivo}

To further investigate the effect of YTHDF1 on breast cancer in vivo, MCF-7 cells stably transduced with a lentiviral vector containing negative control sequence or sh-YTHDF1\#2 sequence were subcutaneously implanted into NOD/SCID mice. By calculating the tumor volume every 7 days, we found that the tumor volume in the sh-YTHDF1\#2 group was significantly lower than that in the sh-NC control group since day28 (Fig. 4A). On day 49 , the mice were killed to measure the tumor weight, and it was found that the tumor weight of mice in the sh-YTHDF1\#2 group was significantly lower than that in the sh-NC group (Fig. 4B, C). Western blot analysis and cell cycle assay of the tumor cells from xenograft showed that YTHDF1 silence could inhibit EMT in vivo and induce G0/G1 phase cell cycle arrest of breast cancer cells (Fig. 4D, E). Furthermore, in the lung metastasis assay, the number of metastatic lung nodules in the sh-YTHDF1\#2 group was significantly less than that in the sh-NC group (Fig. 4F). Collectively, the above results imply that YTHDF1 could promote breast cell proliferative and invasive capacity both in vitro and in vivo. At the same time, the knockdown of YTHDF1 can significantly inhibit EMT in breast cancer. 
A

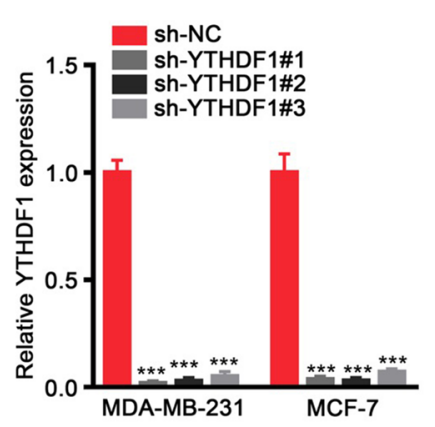

D

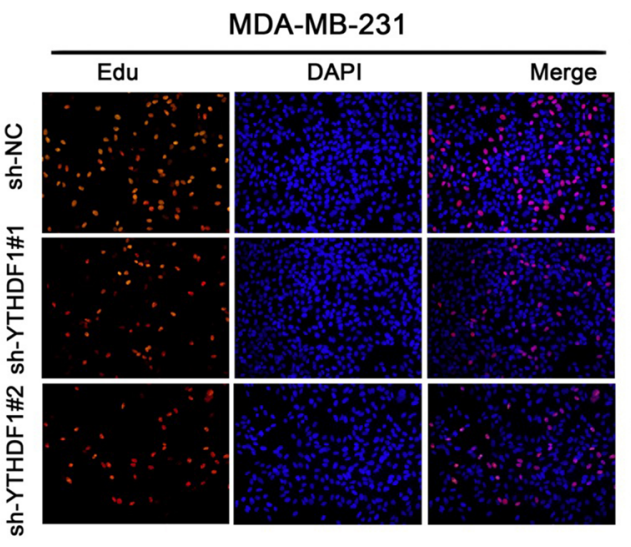

E
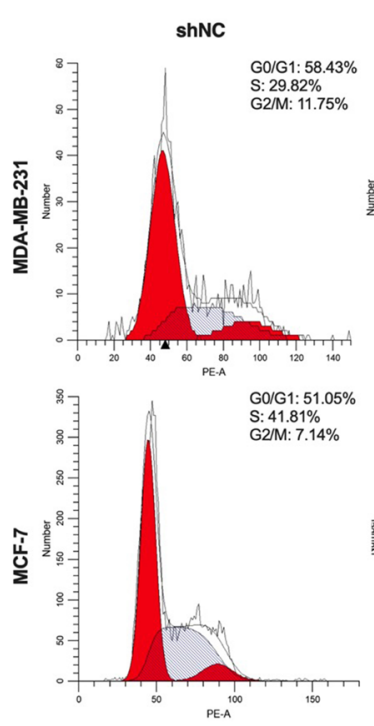

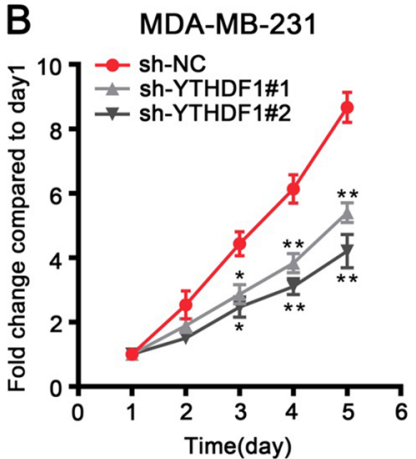

C MCF-7
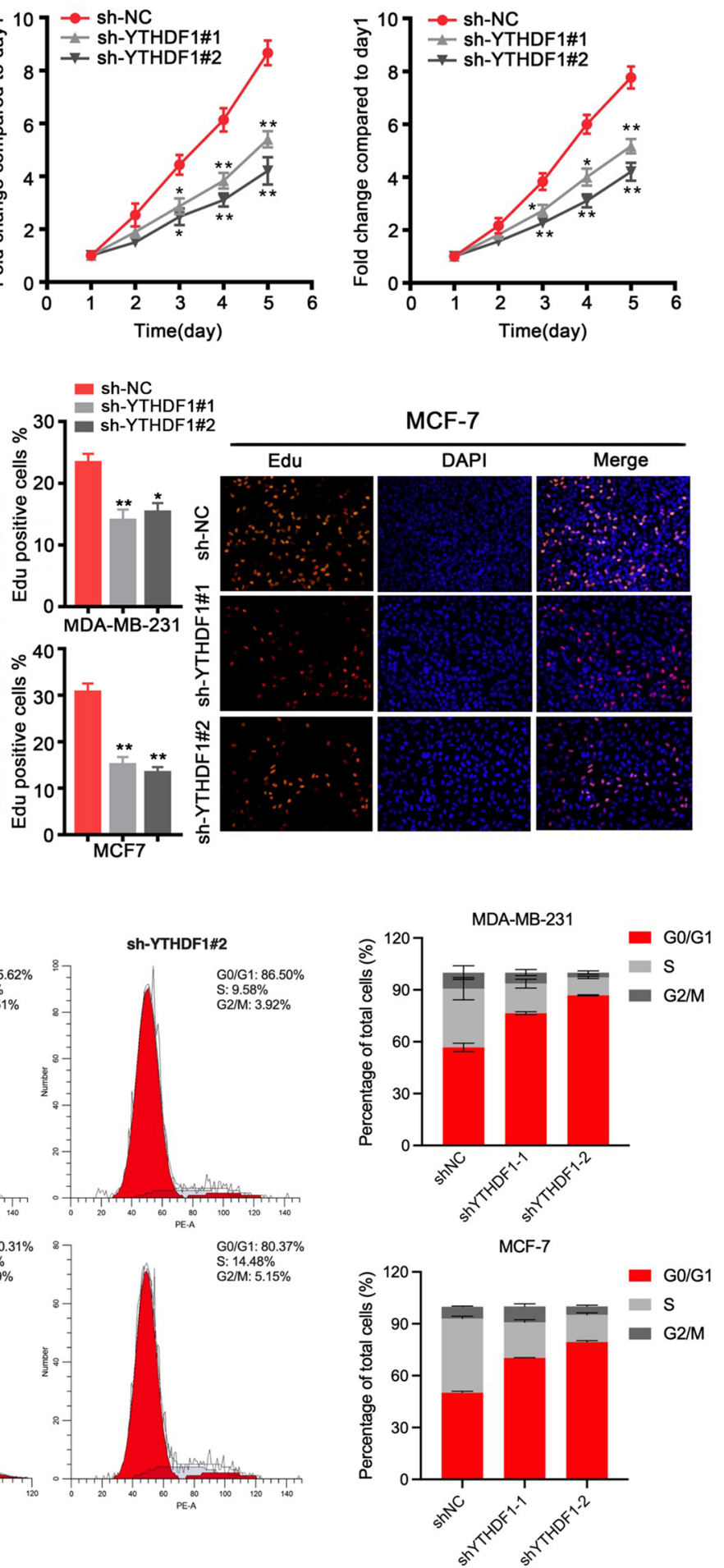

Fig. 2 YTHDF1 knockdown inhibits the growth ability and arrests the cell cycle of breast cancer cells. A Relative YTHDF1 mRNA expression levels after transducing scramble shRNA (sh-NC), sh-YTHDF1\#1, sh-YTHDF1\#2 or sh-YTHDF1\#3 in MDA-MB-231 or MCF-7 cells. B CCK-8 assay, C EdU proliferation assay and $\mathbf{D}$ cell cycle analysis in MDB-MB-231 or MCF-7 cells transduced with sh-NC, sh-YTHDF1\#1 or sh-YTHDF1\#2. All data were presented as means \pm SD of at least three independent repetitions. Values are significant at ${ }^{*} P<0.05,{ }^{* *} P<0.01,{ }^{* * *} P<0.001$ as indicated 


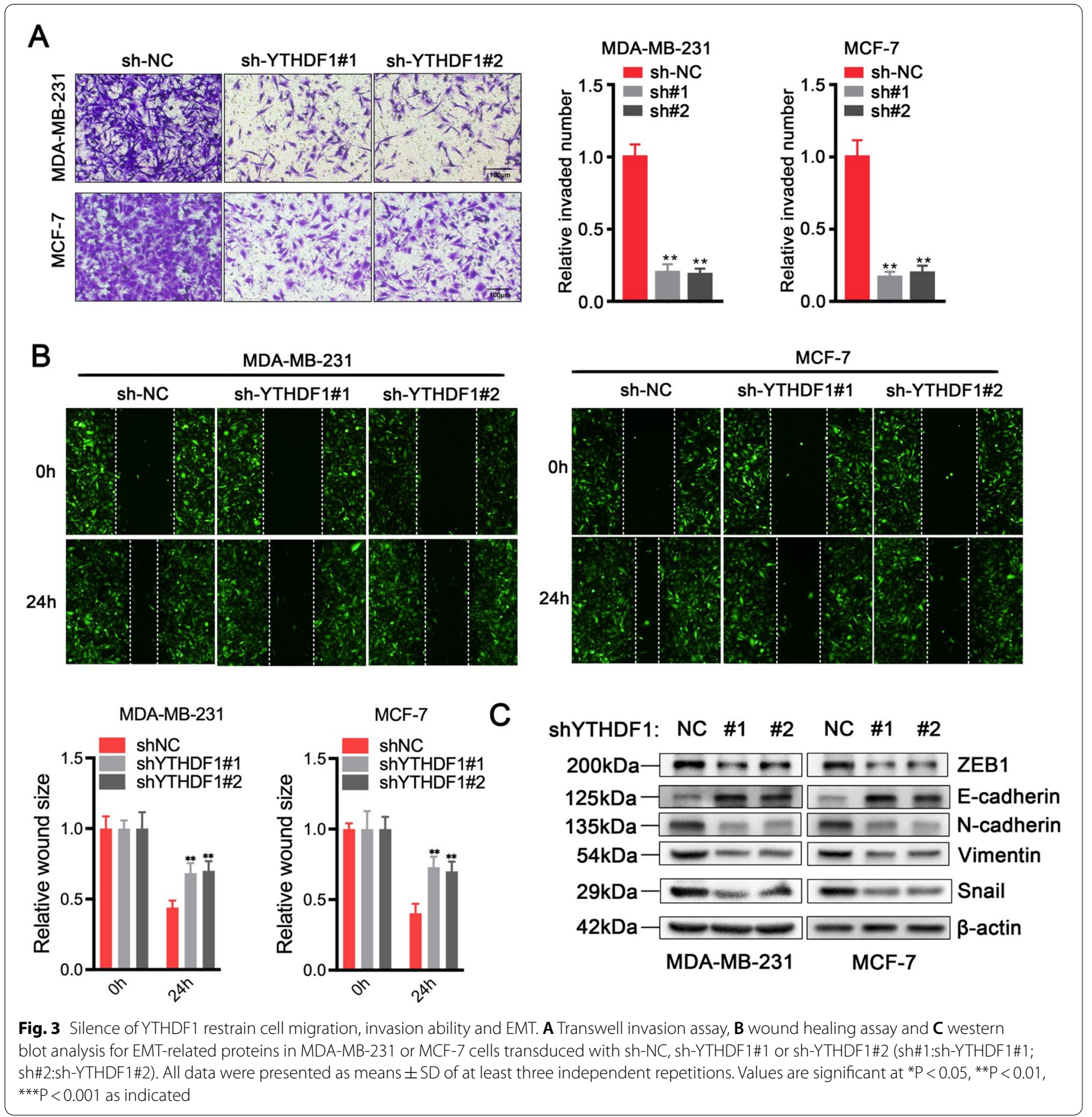

\section{FOXM1 is a direct target of YTHDF1}

YTHDF1 was reported to influence the translation process of the target genes with $\mathrm{m}^{6} \mathrm{~A}$ modifications. Therefore, we used the cBioPortal tool to find the genes whose protein expression was correlated with YTHDF1 mRNA expression in the TCGA-BRCA dataset. Finally, we selected 12 genes as the targets for further study (8 genes with the highest Spearman's correlation coefficients (CCNB1, ENY2, SRC, FOXM1, CCNE1, CCNE2, ASNS and EIF4BP1) and 4 genes that were previously reported to be regulated by YTHDF1 (FZD5, FZD7, FZD9 and WNT5A). After RIP, $\mathrm{m}^{6} \mathrm{~A}-\mathrm{IP}$ and CLIP assays were performed on MCF-7 cells, RT-qPCR was used to detect the expression levels of target genes. We found that FOXM1 and CCNB1 were occupied by YTHDF1 and modified by $\mathrm{m}^{6} \mathrm{~A}$ simultaneously (Fig. 5A-C). Since FOXM1 plays a vital role in EMT, we chose FOXM1. We validated the correlation between YTHDF1 and FOXM1 expression 
A

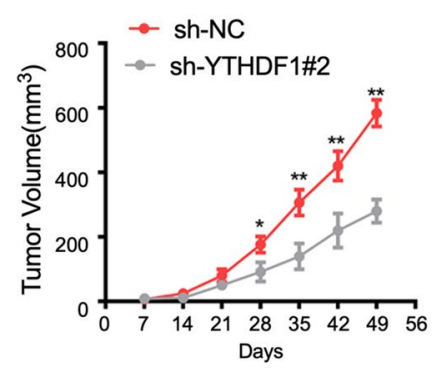

D

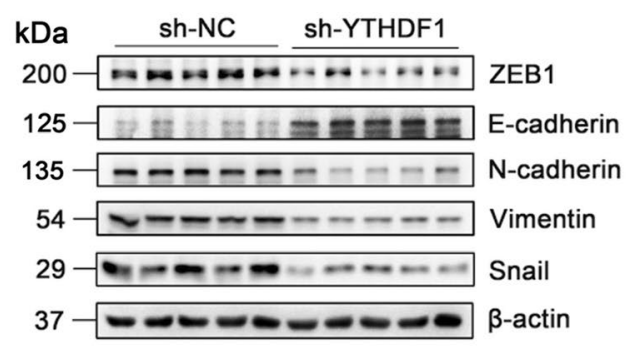

B

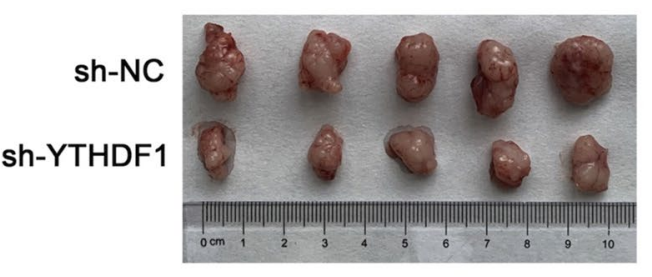

C

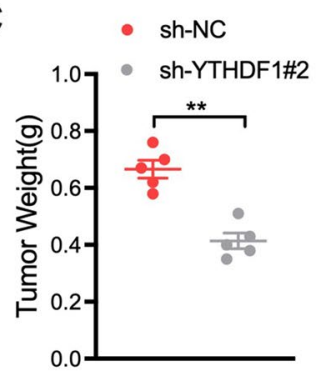

E

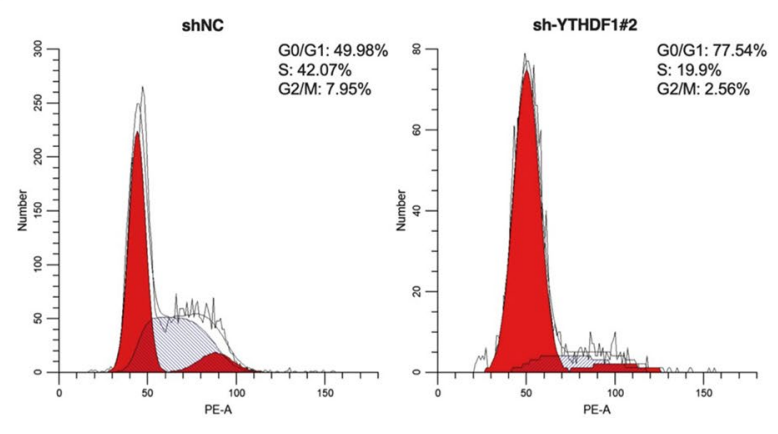

$\mathbf{F}$
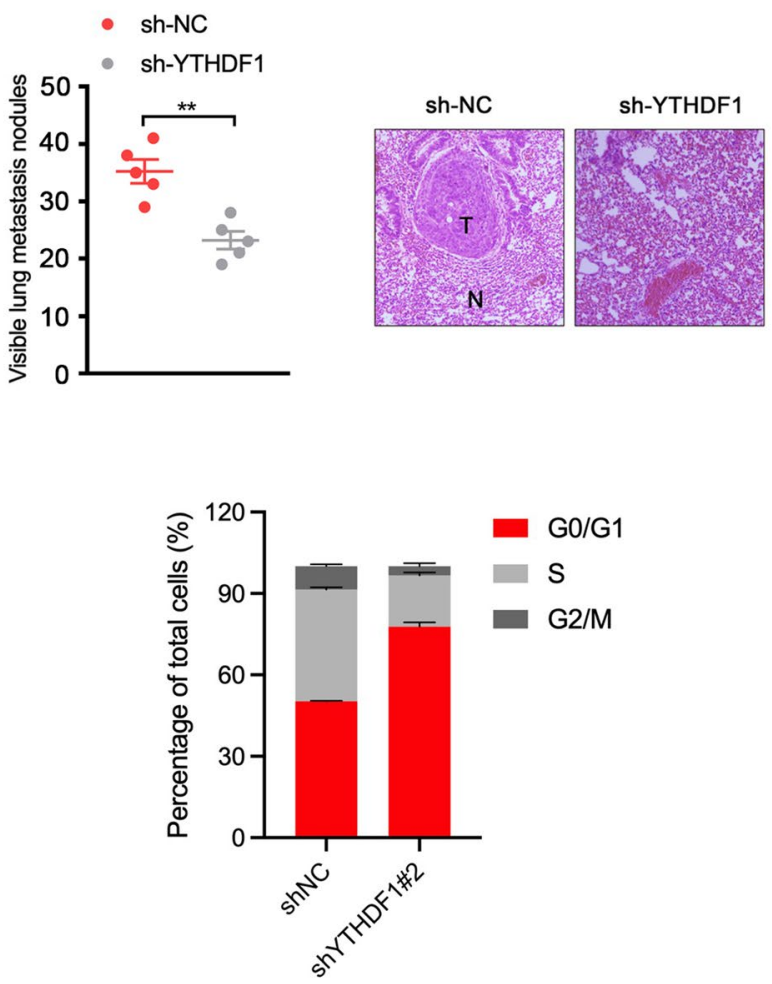

Fig. 4 Depletion of YTHDF1 inhibits the growth of breast cancer cells in vivo. NOD/SCID mice (5 per group) were transplanted with MCF-7 cells $\left(2{ }^{*} 10^{6}\right)$ transduced with sh-NC or sh-YTHDF1\#2. A Tumor volumes in sh-NC or sh-YTHDF1\#2 group at indicated days. B Representative images and $\mathbf{C}$ the weight of tumors in sh-NC or sh-YTHDF1\#2 group at day 49. D The expression of EMT-related proteins and $\mathbf{E}$ cell cycle analysis in xenograft that transduced with sh-NC or sh-YTHDF1. F The number of visible lung metastasis nodules and representative images of lung HE staining slices. ( $T$ stands for metastasis and $\mathrm{N}$ for normal lung tissues). All data were presented as means \pm SD of at least three independent repetitions. Values are significant at ${ }^{*} \mathrm{P}<0.05,{ }^{*} \mathrm{P}<0.01,{ }^{* *} \mathrm{P}<0.001$ as indicated

in breast cancer patients from TCGA by estimating the Pearson's correlation coefficient $\mathrm{R}$. The result indicated a positive correlation $(\mathrm{r}=0.31, P=2.96 \mathrm{e}-29)$ between YTHDF1 and FOXM1 expression (Fig. 5D). In addition, we found that FOXM1 protein expression was highly expressed in breast cancer tissues in The Human Protein Atlas database (Fig. 5E). To study the correlation between YTHDF1 and FOXM1 in protein levels, immunohistochemical analysis was carried out in 9 samples. Through linear regression analysis, it was found that FOXM1 protein levels were positively correlated with YTHDF1, with a correlation coefficient $R^{2}=0.68$ (Fig. 5G).

To explore whether YTHDF1 regulates FOXM1 expression and from which aspect to regulate its expression, RT-qPCR, western blot and polysome profiling analysis were performed on breast cancer cells transduced with sh-NC or sh-YTHDF1\#2. The results showed that for FOXM1, there was no significant difference in the mRNA expression between the sh-NC and sh-YTHDF1\#2 groups. However, a considerable decrease in the protein 


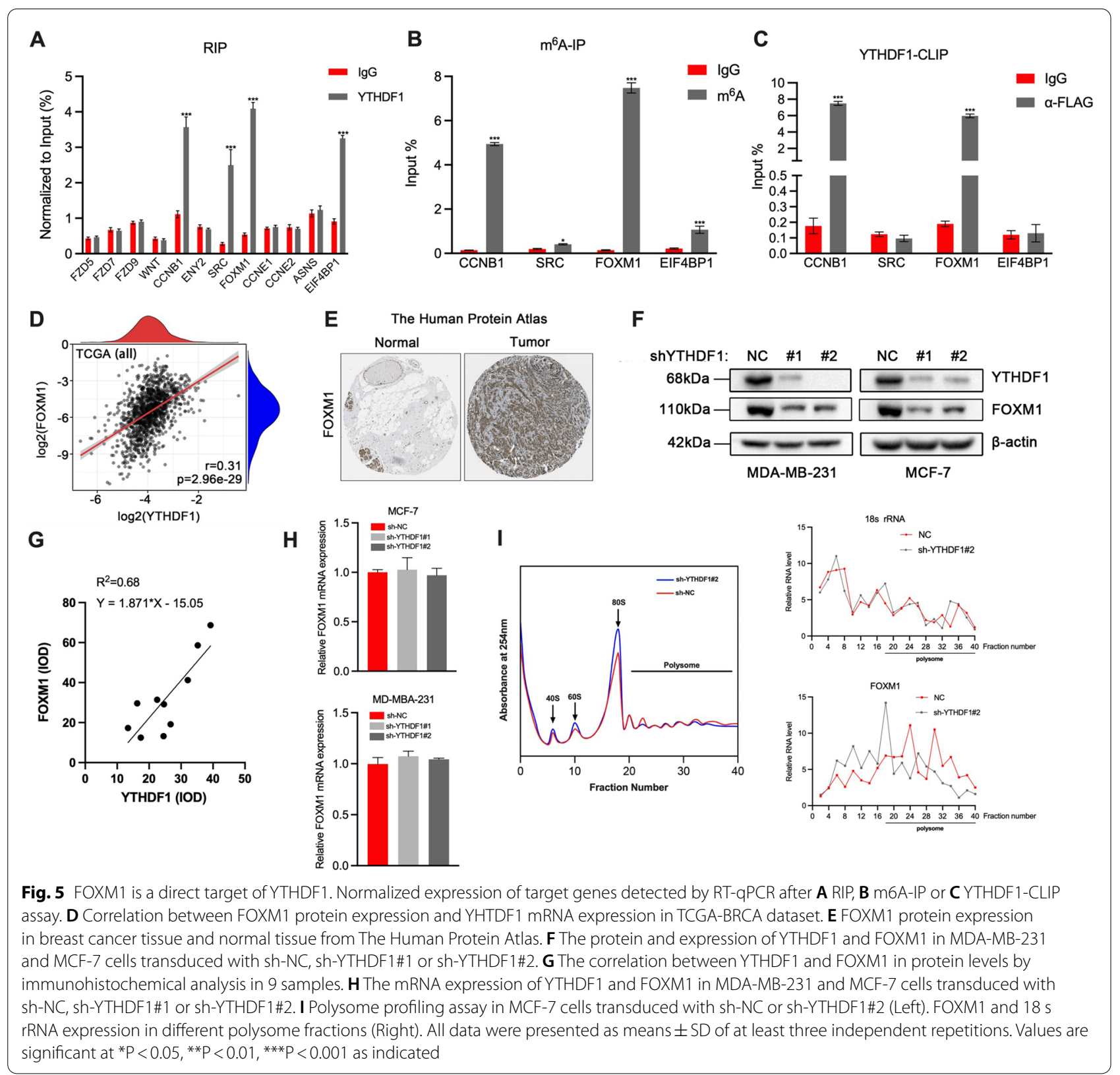

expression was observed in the sh-YTHDF1\#2 group (Fig. 5F, H). As shown in Fig. 5I, inhibition of YTHDF1 slightly decreased the translation level of MCF-7 cells. However, YTHDF1 knockdown inhibited FOXM1 translation significantly more than $18 \mathrm{~S}$ rRNA. We further conducted polysome profiling assays in MCF-7 cells transfected with YTHDF1-WT and YTHDF1-MUT plasmids. As shown in Additional file 4: Fig. S4, the translational level of YTHDF1 increased in YTHDF1WT but not in YTHDF1-MUT MCF-7 cells, indicating that mutant YTHDF1 did not promote FOXM1 translation. These results suggest that as a target of YTHDF1,
FOXM1 is not regulated by YTHDF1 via affecting its transcription but promoting FOXM1 translation in breast cancer cells.

\section{YTHDF1 regulates FOXM1 expression in an m6A-dependent manner}

Since YTHDF1 can specifically identify the m6A modification and are responsible for the outcomes of its target genes, we then try to explore whether YTHDF1 promotes FOXM1 translation in an $\mathrm{m}^{6} \mathrm{~A}$ dependent manner. Mutations of the YTH domain were introduced into the YTHDF1-WT plasmid to construct the 
YTHDF1-MUT plasmid (Additional file 2: Fig. S2A) $[11,25,26]$. As shown in Fig. 6A, B, an anti-Flag antibody was used for RIP assay, followed by RT-qPCR and western blot analysis to detect the expression of FOXM1. The results suggested that the binding of FOXM1 to YTHDF1 was significantly reduced in MDMBA-231 and MCF-7 cells transfected with YTHDF1MUT plasmids. Then we introduced mutations into the m6A motif of the FOXM1-WT plasmid to construct the FOXM1-MUT plasmid (Fig. 6C, Additional file 2: Fig. S2B). Subsequently, FOXM1-WT and FOXM1-MUT plasmids were co-transfected into the breast cancer cells with Empty vector, YTHDF1-WT and YTHDF1MUT plasmids, respectively. The results suggested that the expression of FOXM1 was significantly increased only in the cells co-transfected with YTHDF1-WT and FOXM1-WT plasmids, while FOXM1-MUT had no response to the overexpression of YTHDF1-WT (Fig. 6D). In summary, these results confirmed that YTHDF1 modulated the translation process of FOXM1 in an $\mathrm{m}^{6} \mathrm{~A}$ dependent manner.

\section{The inhibited effects of YTHDF1 silence could be rescued by FOXM1 overexpression}

We further verified the role of the YTHDF1/FOXM1 axis in breast cancer progression. Plasmids that contain the FOXM1 sequence (FOXM1) were transfected into the sh-YTHDF\#2 MDA-MB-231 and MCF-7 cells, respectively. Western blot analysis was used to verify the transfection efficiency (Fig. 7A). Subsequently, CCK-8 assay, transwell, and wound healing assay were performed on breast cancer cells with sh-NC, sh-YTHDF1\#2 or shYTHDF1\#2+FOXM1, respectively. The results showed that depletion of YTHDF1 inhibited tumor proliferation, migration, and invasion, which was partially abolished by overexpression of FOXM1 (Fig. 7B-D). Consistent with these results, the western blot assay validated that sh-YTHDF1\#2+FOXM1 cells could counteract the changes of epithelial and mesenchymal markers induced by YTHDF1 silence (Fig. 7E).

To further confirm that YTHDF1 regulates cell growth through FOXM1, we transfected FOXM1-MUT plasmid into sh-YTHDF1\#2 MCF-7 cells and found that the

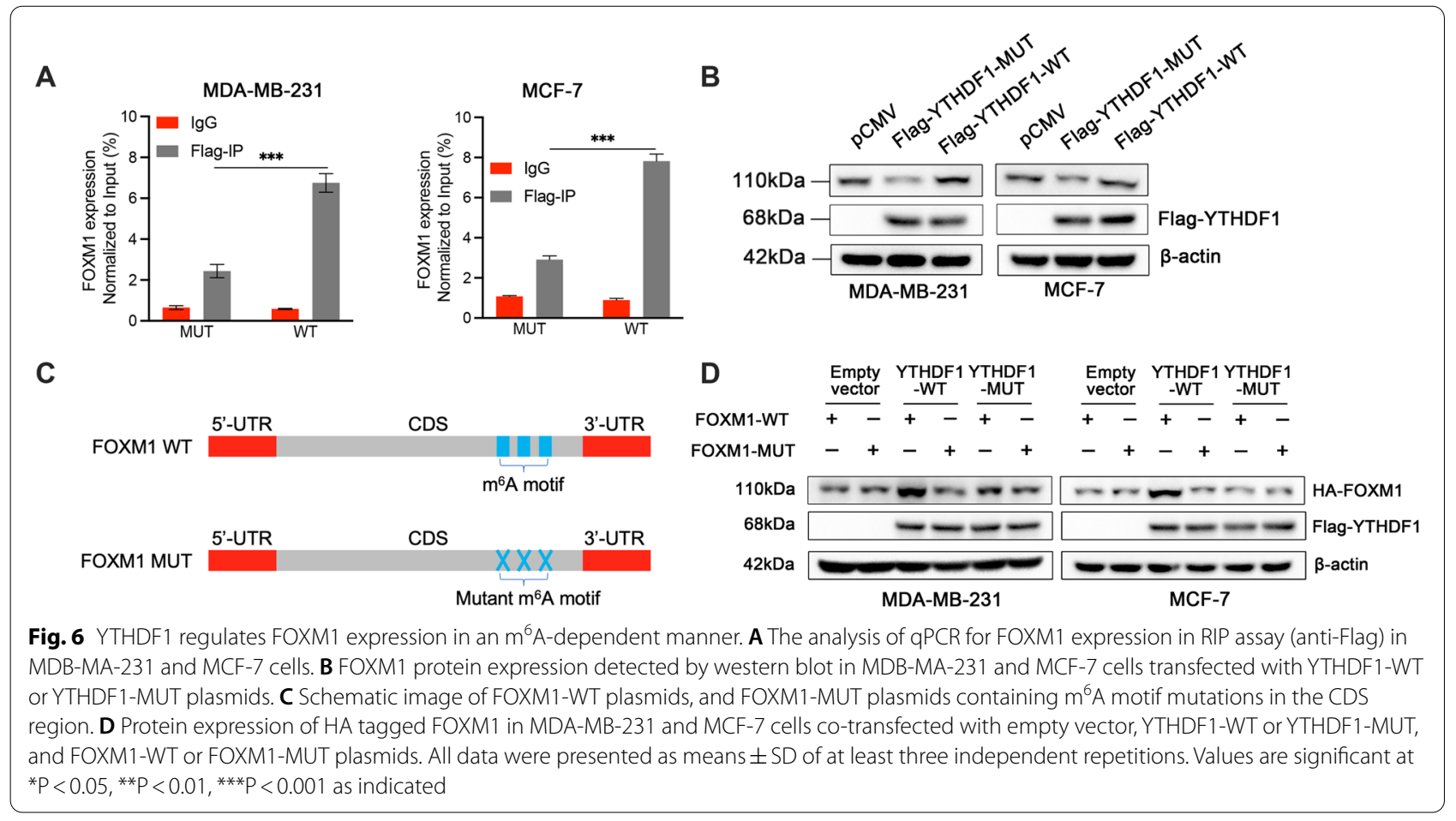

(See figure on next page.)

Fig. 7 The anti-tumor effect of YTHDF1 silence could be abrogated by FOXM1 overexpression. A Western blot analysis for FOXM1 and YTHDF1 in MDB-MA-231 and MCF-7 cells with sh-YTHDF1-2 (shYTH) or sh-YTHDF1\#2 + pcDNA-FOXM1 plasmid (shYTH + FOXM1) B CCK-8 assay, C Transwell invasion assay, $\mathbf{D}$ wound healing assay and $\mathbf{E}$ Western blot assay for EMT-related proteins in MDB-MA-231 and MCF-7 cells with shNC, sh-YTHDF1\#2 (shYTH) or sh-YTHDF1\#2 + FOXM1 (shYTH + FOXM1). All data were presented as means \pm SD of at least three independent repetitions. Values are significant at ${ }^{*} P<0.05,{ }^{*} P<0.01,{ }^{* *} P<0.001$ as indicated 


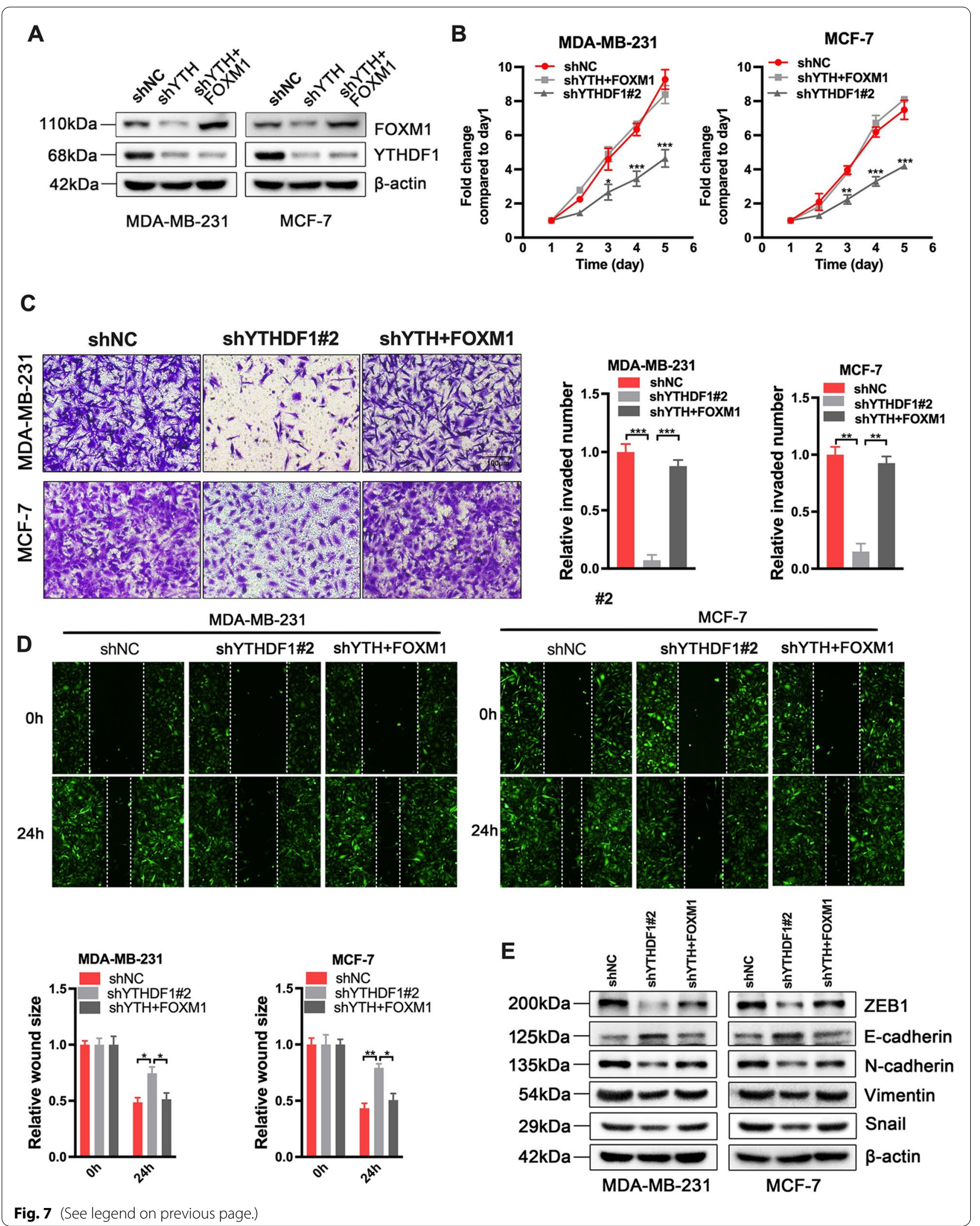



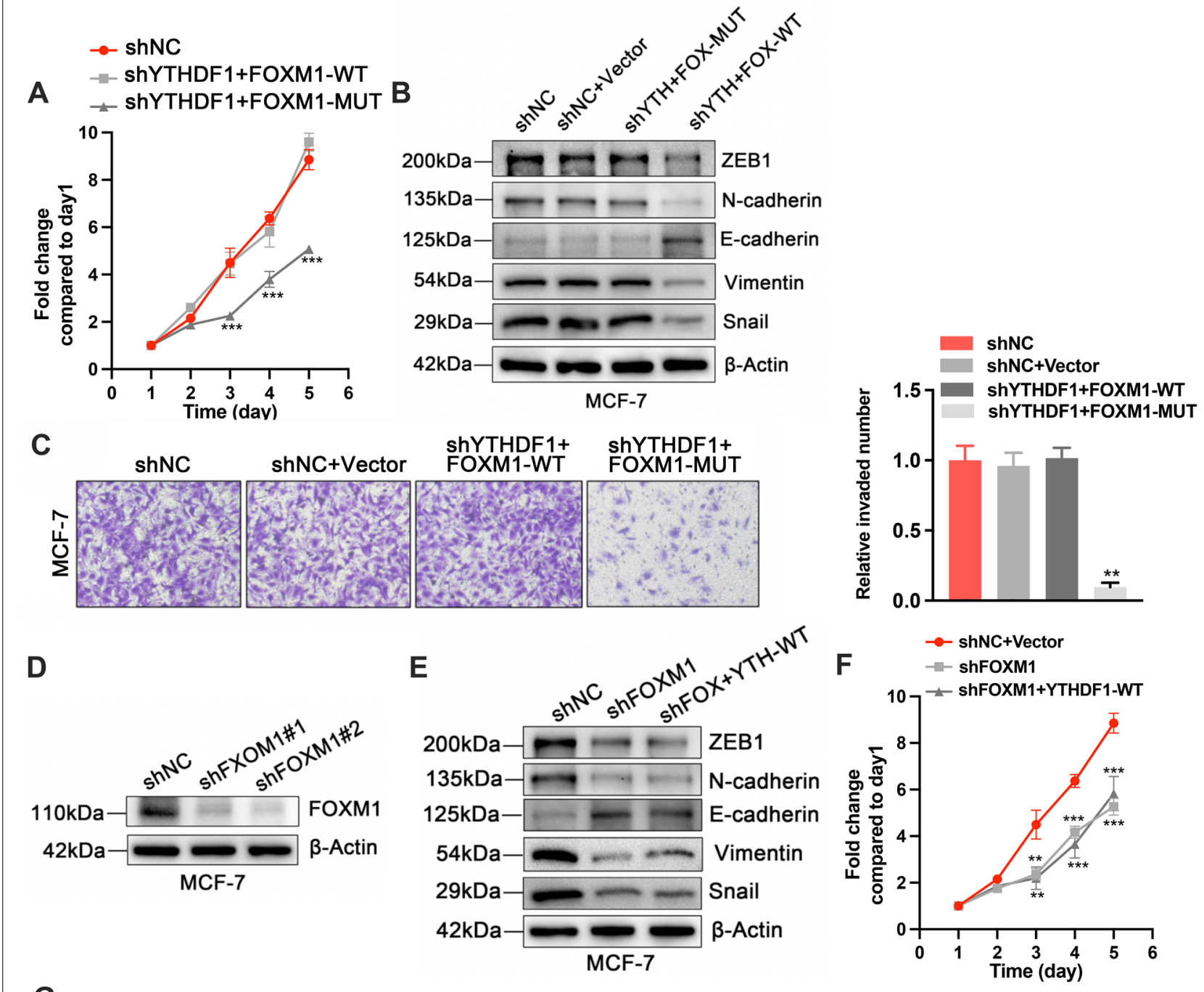

G

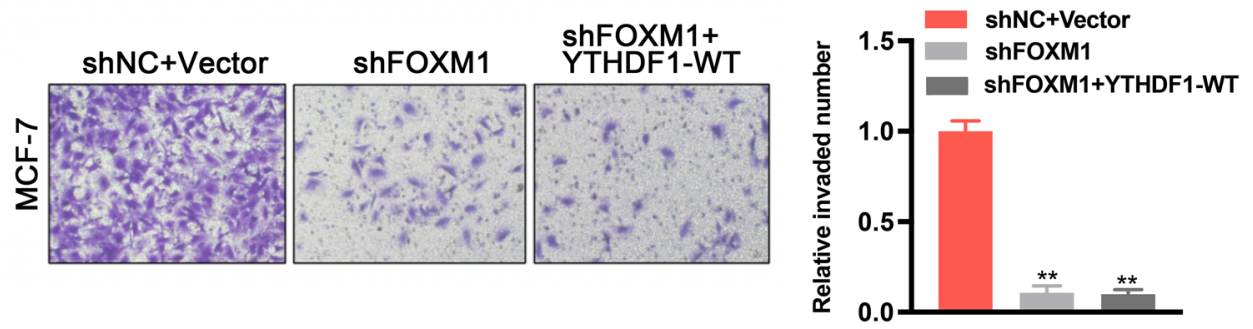

Fig. 8 YTHDF1 overexpression could not reverse the anti-tumor effects caused by FOXM1 knockdown in MCF-7 cells. A CCK-8 assays in shNC, shYTHDF1\#2 + FOXM1-WT and sh-YTHDF1\#2 + FOXM1-MUT MCF-7 cells. B Western blots for EMT-related proteins and C Transwell invasion assays in shNC, shNC + Vector, sh-YTHDF1\#2 + FOXM1-MUT and sh-YTHDF1\#2+FOXM1-WT MCF-7 cells. D FOXM1 protein expression in shNC, sh-FOXM1\#1 and sh-FOXM1\#2 transduced cells. E Western blots for EMT-related proteins, F CCK-8 assays and $\mathbf{G}$ Transwell invasion assays in shNC+Vector, sh-YTHDF1\#2 + FOXM1-MUT and sh-YTHDF1\#2+FOXM1-WT MCF-7 cells. All data were presented as means \pm SD of at least three independent repetitions. Values are significant at ${ }^{*} \mathrm{P}<0.05$, ${ }^{* *} \mathrm{P}<0.01$, ${ }^{* *} \mathrm{P}<0.001$ as indicated 
mutant FOXM1 could not reverse the cell growth inhibition induced by YTHDF1 knockdown (Fig. 8A-C). The result confirmed that FOXM1 was downstream of YTHDF1. In addition, shRNAs were designed and transduced into MCF-7 cells to knock down FOXM1 expression (Fig. 8D). Through CCK-8, Transwell invasion and Western blot assays, we found that FOXM1 knockdown significantly inhibited cell growth, invasion and EMT. Besides, in FOXM1 knockdown MCF-7 cells, overexpression of YTHDF1 could not reverse the inhibitory effects on cell growth caused by FOXM1 knockdown (Fig. 8E-G).

Taken together, these data suggested that YTHDF1 knockdown blocks the proliferation, migration, and invasion of breast cancer cells, but that could be rescued by FOXM1 overexpression simultaneously.

\section{Discussion}

$\mathrm{m}^{6} \mathrm{~A}$ modification abnormalities have been shown to play critical roles in developing various tumors and are associated with the persistence of tumor stem cells [1]. In recent years, a study confirmed that YTHDF3 promotes breast cancer metastasis by promoting the translation of ST6GALNAC5, GJA1 and EGFR [27]. Another study demonstrated the demethylase FTO promotes breast cancer progression by inhibiting BNIP3 [28]. Ramesh et al. reported that high expression of YTHDF1 and TYHDF3 was closely associated with breast cancer metastasis and poor prognosis [21]. Despite the prognostic role of m6A "reader" YTHDF1 mentioned in the above article, however, the biomechanism of YTHDF1 involved in the carcinogenesis and metastasis of breast cancer remains unclear. Thus, it is urgent to elucidate the underlying mechanisms in breast cancer progression and discover reliable biomarkers to predict prognosis.

As an $\mathrm{m}^{6} \mathrm{~A}$ reading protein, YTHDF1 promotes translation by recognizing and binding to the $\mathrm{m}^{6} \mathrm{~A}$ modified mRNAs [29]. Several studies have explored its function. YTHDF1 could act as an oncogene or a tumor suppressor gene in tumors. YTHDF1 promoted the eIF3C translation in ovarian cancer, thus promoting the development and metastasis of ovarian cancer [25]. In lung cancer, YTHDF1 increased the translation of the YAP gene and induced metastasis and drug resistance in lung cancer cells [30]. In hepatocellular carcinoma (HCC), higher expression of YTHDF1 was associated with advanced stages and unfavorable prognosis [19]. Bioinformatic analysis indicated that in HCC, the expression of YTHDF1 was related to the expression of cell cyclerelated genes. In addition, YTHDF1 promoted HCC EMT by upregulating Snail [31]. In colorectal cancer (CRC) cells, YTHDF1 induced cisplatin resistance by regulating glutamine metabolism through glutaminase [17].
In contrast to the tumor-promoting effects of YTHDF1 in the above tumors, YTHDF1 inhibited melanoma progression by promoting the translation of the tumor suppressor gene HINT2 [32].

In the present study, we found that the expression of YTHDF1 in breast cancer samples and breast cancer cell lines was significantly higher than that of the normal controls, and its high expression was significantly correlated with the shortened OS, larger tumor size, as well as positive lymphatic metastasis and distant metastasis. We explored the expression of YTHDF1 in different subtypes of breast cancer and did not find that it was significantly increased or decreased in the HER2+ subtype. However, its high expression predicted poor prognosis of HER2+ patients, suggesting that it might play an important role in helping to continuously activate HER2+ downstream signaling pathways (such as PI3K/ AKT pathway) or alternative pathways (IGF-IR pathway) to induce resistance to HER2+ targeted therapy. After YTHDF1 was knocked down by shRNA in MCF-7 and MDA-MB-231 cells, the proliferation, migration, invasion and EMT of the tumor cells were significantly suppressed, and G0/G1 phase cell cycle arrest occurred. Subsequently, we confirmed that FOXM1 was a direct target of YTHDF1 through RIP, $\mathrm{m}^{6} \mathrm{~A}-\mathrm{IP}, \mathrm{YTHDF} 1-\mathrm{CLIP}$ and polysome profiling analysis. YTHDF1 regulated FOXM1 mainly by facilitating its translation, which was implemented in a manner dependent on $\mathrm{m}^{6} \mathrm{~A}$.

EMT was considered an essential step in tumor metastasis [33], and its occurrence represents the transformation of differentiated epidermal cells into mesenchymal cancer cells [34]. It also plays a vital role in the occurrence, development and metastasis of breast cancer [35]. FOXM1 was a crucial gene that promoted EMT in a variety of tumors [36-40]. In breast cancer, FOXM1 was shown to promote EMT by facilitating Slug gene transcription [41]. FOXM1 expression and activity were known to be regulated in diverse ways. In breast cancer, HMGA1 [42], EGF [43, 44] and TGF $\beta[45,46]$ signaling pathways, HIF-1 $\alpha$ [47] all promoted EMT by upregulating FOXM1. In addition, FOXM1 could be epigenetic regulated [48] or deubiquitinated by USP21 [49] or OTUB1 [50]. In this study, we described a novel regulatory mechanism of FOXM1. That is, FOXM1 was modulated by YTHDF1 in an $\mathrm{m}^{6} \mathrm{~A}$-dependent manner. In addition, a previous study reported that FOXM1 not only promoted breast cancer progression and metastasis through EMT but also mediated PI3K $\alpha$ inhibitor resistance in ER+ breast cancer [51].

Currently, most studies on YTHDF1 focused on regulating its target genes. Only a few studies have explored the regulation of YTHDF1 expression. For example, Hypoxia was found to upregulate YTHDF1 expression 
in a HIF-1 $\alpha$ dependent manner in hepatocellular carcinoma [52]. c-Myc promoted the expression of YTHDF1 in colorectal carcinoma [53]. In addition, the Wnt/ $\beta$-catenin signaling pathway [54], the m6a "eraser" ALKBH5 [55], and miR-3436 [56] were reported to regulate YTHDF1 expression in cancers. The regulatory mechanisms of YTHDF1 are complex. We will carry out further studies to explore the upstream of YTHDF1.

\section{Conclusion}

We investigated the specific biological role of YTHDF1 in breast cancer, and our results revealed that YTHDF1 was aberrantly overexpressed and significantly associated with unfavorable survival rates of breast cancer. Moreover, we demonstrated that YTHDF1 played a crucial role in promoting cell proliferation and invasion in cancer cells via the YTHDF1/FOXM1 axis, aggravating breast cancer progression and metastasis consequently. Collectively, our study proposed a novel regulatory pathway of YTHDF1, which provided a new idea for breast cancer treatment and suggested that YTHDF1 may be a potential biomarker and therapeutic target for breast cancer patients.

\section{Abbreviations}

$m^{6} \mathrm{~A}$ : N6-methyladenosine; EMT: Epithelial-mesenchymal transformation; mRNA: Messenger RNA; CDS: Coding sequence; HE: Hematoxylin and eosin; CLIP: Cross-linking immunoprecipitation; m6A-IP: M6A immunoprecipitation; BRCA: Breast invasive carcinoma; OS: Overall survival; YTHDF1:YTH N6-methyladenosine RNA binding protein 1.

\section{Supplementary Information}

The online version contains supplementary material available at https://doi. org/10.1186/s13578-022-00759-w.

Additional file 1: Figure S1. (A) The expression of YTHDF1 in breast cancer patients of different stages. (B) Expression of YTHDF1 in breast cancer tissues based on breast cancer subclasses in the TCGA-BRCA dataset. The effects of YTHDF1 on overall survival (OS) in (C) Luminal, (D) HER2+ and (E) Basel-like breast cancer.

Additional file 2: Figure S2. Plasmid information of YTHDF1 and FOXM1. (A) Schematic image of YTHDF1-WT and YTHDF1-MUT plasmids (Flagtagged). (B) The detailed mutation information of $\mathrm{m}^{6} \mathrm{~A}$ motif in FOXM1MUT plasmids.

Additional file 3: Figure S3. YTHDF1 knockdown by siRNAs inhibits the growth and invasive ability of MCF-7 cells. (A) Relative YTHDF1 mRNA expression levels after transfecting scramble si-NC, si-YTHDF1-1, si-YTHDF1-2 in MCF-7 cells. (B) CCK-8 assays, (C) Transwell invasion assays, (D) EdU proliferation assays and (E) Western blots for EMT-realted proteins in MCF-7 cells transfected with si-NC, si-YTHDF1-1 or si-YTHDF1-2. All data were presented as means $\pm \mathrm{SD}$ of at least three independent repetitions. Values are significant at ${ }^{*} \mathrm{P}<0.05,{ }^{* *} \mathrm{P}<0.01,{ }^{* * *} \mathrm{P}<0.001$ as indicated.

Additional file 4: Figure S4. Polysome profiling assay in MCF-7 cells transduced with vector and YTHDF1-WT (Left) or YTHDF1-MUT (Right).

Additional file 5: Table S1. Primers for RT-qPCR. Table S2. Clinicopathological correlations of YTHDF1 expression in breast cancer.
Acknowledgements

Not applicable.

\section{Authors' contributions}

$H C, Y Y, M Y$ and $H H$ designed and performed the experiments; JH, ZX, SM, LY and $H G$ collected and validate the clinical samples data; $X H, H W, G Y$ and $G T$ extracted, analyzed and interpreted the data from GEO and TCGA databases. WZ, FZ and HH wrote the manuscript. GT and LL made substantial contributions to the conception of the work and substantively revised it. HW, WZ and LL contributed to the study supervision. All authors read and approved the final manuscript.

\section{Funding}

This study was supported by the National Natural Science Foundation of China (NSFC) (Grant number: No. 81702397, 81860529 and 81860650). The study was supported by the Hainan Provincial Key Research and Development Program Project Fund (No. ZDYF2021SHFZ248). This project supported by Hainan Province Clinical Medical Center.

\section{Availability of data and materials}

All data created or analyzed during this study are enrolled in this published article or are available from the corresponding author on reasonable request.

\section{Declarations}

\section{Ethics approval and consent to participate}

This project was approved by the Ethics Committee of Union Hospital, Tongji Medical College, Huazhong University of Science and Technology.

\section{Consent for publication}

Not applicable.

\section{Competing interests}

The authors declare that they have no competing interests.

\section{Author details}

'Department of Breast and Thyroid Surgery, The Second Affiliated Hospital of Hainan Medical University, Key Laboratory of Tropical Translational Medicine of Ministry of Education \& Hainan Provincial Key Laboratory of Tropical Medicine, Haikou 570311, China. ${ }^{2}$ Department of Breast and Thyroid Surgery, Union Hospital, Tongji Medical College, Huazhong University of Science and Technology, Wuhan 430022, China. ${ }^{3}$ Department of Pancreatic Surgery, Union Hospital, Tongji Medical College, Huazhong University of Science and Technology, Wuhan 430022, China. ${ }^{4}$ Department of Neurosurgery, General Hospital of Central Theater Command of Chinese People's Liberation Army, Wuhan 430070, China. ${ }^{5}$ Medical College Wuhan University of Science and Technology, Wuhan 430070, China. ${ }^{6}$ Department of Emergency Medicine, Affiliated Hospital of Sergeant School Affiliated to Army Medical University, Shijiazhuang 050047, China.

Received: 17 September 2021 Accepted: 10 February 2022 Published online: 23 February 2022

\section{References}

1. Fu Y, et al. Gene expression regulation mediated through reversible $\mathrm{m}^{6} \mathrm{~A}$ RNA methylation. Nat Rev Genet. 2014;15(5):293-306.

2. Zaccara S, Ries RJ, Jaffrey SR. Reading, writing and erasing mRNA methylation. Nat Rev Mol Cell Biol. 2019;20(10):608-24.

3. Huang $\mathrm{H}$, Weng $\mathrm{H}$, Chen J. m(6)A modification in coding and noncoding RNAs: Roles and therapeutic implications in cancer. Cancer Cell. 2020;37(3):270-88.

4. Ma Z, Ji J. N6-methyladenosine (m6A) RNA modification in cancer stem cells. Stem Cells. 2020;38(12):1511-9.

5. Zhang $\mathrm{Y}$, et al. m6A modification in RNA: biogenesis, functions and roles in gliomas. J Exp Clin Cancer Res. 2020;39(1):192.

6. Wang $Y$, et al. Emerging roles of N6-methyladenosine $(\mathrm{m}(6) \mathrm{A})$ modification in breast cancer. Cell Biosci. 2020;10(1):136. 
7. Fang R, Ye L, Shi H. Understanding the roles of N(6)-methyladenosine writers, readers and erasers in breast cancer. Neoplasia. 2021;23(6):551-60.

8. Zheng F, et al. The emerging role of RNA N6-methyladenosine methylation in breast cancer. Biomark Res. 2021;9(1):39.

9. Dominissini $\mathrm{D}$, et al. Topology of the human and mouse m6A RNA methylomes revealed by m6A-seq. Nature. 2012;485(7397):201-6.

10. Wang $X$, et al. N6-methyladenosine-dependent regulation of messenger RNA stability. Nature. 2014:505(7481):117-20.

11. Xu C, et al. Structural basis for the discriminative recognition of n6-methyladenosine RNA by the human YT521-B homology domain family of proteins. J Biol Chem. 2015;290(41):24902-13.

12. Imam H, et al. Interferon-stimulated gene 20 (ISG20) selectively degrades N6-methyladenosine modified hepatitis B virus transcripts. PLoS Pathog. 2020;16(2):e1008338.

13. Bai Y, et al. YTHDF1 regulates tumorigenicity and cancer stem cell-like activity in human colorectal carcinoma. Front Oncol. 2019;9:332.

14. Bushkin GG, et al. m(6)A modification of a 3' UTR site reduces RME1 mRNA levels to promote meiosis. Nat Commun. 2019;10(1):3414.

15. Wang $X$, et al. $N(6)$-methyladenosine modulates messenger RNA translation efficiency. Cell. 2015;161(6):1388-99.

16. Liu S, et al. The roles and mechanisms of YTH domain-containing proteins in cancer development and progression. Am J Cancer Res. 2020;10(4):1068-84.

17. Chen P, et al. Targeting YTHDF1 effectively re-sensitizes cisplatin-resistant colon cancer cells by modulating GLS-mediated glutamine metabolism. Mol Ther Oncolytics. 2021;20:228-39.

18. LiT, et al. Increased expression of YTHDF1 and HNRNPA2B1 as potent biomarkers for melanoma: a systematic analysis. Cancer Cell Int. 2020;20:239.

19. Zhao X, et al. Overexpression of YTHDF1 is associated with poor prognosis in patients with hepatocellular carcinoma. Cancer Biomark. 2018;21(4):859-68.

20. Shi Y, et al. YTHDF1 links hypoxia adaptation and non-small cell lung cancer progression. Nat Commun. 2019;10(1):4892.

21. Anita $R$, et al. The m6A readers YTHDF1 and YTHDF3 aberrations associated with metastasis and predict poor prognosis in breast cancer patients. Am J Cancer Res. 2020;10(8):2546-54.

22. Fedchenko N, Reifenrath J. Different approaches for interpretation and reporting of immunohistochemistry analysis results in the bone tissuea review. Diagn Pathol. 2014;9:221-221.

23. Van Nostrand EL, et al. Robust transcriptome-wide discovery of RNAbinding protein binding sites with enhanced CLIP (eCLIP). Nat Methods. 2016;13(6):508-14.

24. Pathria $\mathrm{G}$, et al. Translational reprogramming marks adaptation to asparagine restriction in cancer. Nat Cell Biol. 2019;21(12):1590-603.

25. Liu T, et al. The m6A reader YTHDF1 promotes ovarian cancer progression via augmenting EIF3C translation. Nucleic Acids Res. 2020;48(7):3816-31.

26. Pi J, et al. YTHDF1 promotes gastric carcinogenesis by controlling translation of FZD7. Cancer Res. 2021;81(10):2651-65.

27. Chang $G$, et al. YTHDF3 induces the translation of $m(6) A$-enriched gene transcripts to promote breast cancer brain metastasis. Cancer Cell. 2020;38(6):857-871.e7.

28. Niu Y, et al. RNA N6-methyladenosine demethylase FTO promotes breast tumor progression through inhibiting BNIP3. Mol Cancer. 2019;18(1):46.

29. Lan $Q$, et al. The critical role of RNA $m^{6} A$ methylation in cancer. Can Res. 2019;79(7):1285

30. Jin $D$, et al. $m(6) A$ mRNA methylation initiated by METTL3 directly promotes YAP translation and increases YAP activity by regulating the MALAT1-miR-1914-3p-YAP axis to induce NSCLC drug resistance and metastasis. J Hematol Oncol. 2019:12(1):135.

31. Lin X, et al. RNA m(6)A methylation regulates the epithelial mesenchymal transition of cancer cells and translation of snail. Nat Commun. 2019;10(1):2065

32. Jia $R$, et al. $m(6) A$ modification suppresses ocular melanoma through modulating HINT2 mRNA translation. Mol Cancer. 2019;18(1):161.

33. Tiwari $\mathrm{N}$, et al. EMT as the ultimate survival mechanism of cancer cells. Semin Cancer Biol. 2012;22(3):194-207.

34. Lu W, Kang Y. Epithelial-mesenchymal plasticity in cancer progression and metastasis. Dev Cell. 2019;49(3):361-74.
35. Xue C, et al. The gatekeeper effect of epithelial-mesenchymal transition regulates the frequency of breast cancer metastasis. Cancer Res. 2003;63(12):3386-94.

36. Nilsson MB, et al. A YAP/FOXM1 axis mediates EMT-associated EGFR inhibitor resistance and increased expression of spindle assembly checkpoint components. Sci Transl Med. 2020;12(559):eaaz4589.

37. Zhang $X$, et al. Akt/FoxM1 signaling pathway-mediated upregulation of MYBL2 promotes progression of human glioma. J Exp Clin Cancer Res. 2017:36(1):105.

38. Xing $\mathrm{S}$, et al. Hypoxia downregulated miR-4521 suppresses gastric carcinoma progression through regulation of IGF2 and FOXM1. Mol Cancer. 2021;20(1):9.

39. Lv X, et al. Circ-MMP2 (circ-0039411) induced by FOXM1 promotes the proliferation and migration of lung adenocarcinoma cells in vitro and in vivo. Cell Death Dis. 2020;11(6):426.

40. Li M, et al. Activation of an AKT/FOXM1/STMN1 pathway drives resistance to tyrosine kinase inhibitors in lung cancer. $\mathrm{Br} J$ Cancer. 2017;117(7):974-83

41. Yang $C$, et al. FOXM1 promotes the epithelial to mesenchymal transition by stimulating the transcription of Slug in human breast cancer. Cancer Lett. 2013;340(1):104-12.

42. Zanin R, et al. HMGA1 promotes breast cancer angiogenesis supporting the stability, nuclear localization and transcriptional activity of FOXM1. J Exp Clin Cancer Res. 2019:38(1):313.

43. Lo HW, et al. Epidermal growth factor receptor cooperates with signal transducer and activator of transcription 3 to induce epithelial-mesenchymal transition in cancer cells via up-regulation of TWIST gene expression. Cancer Res. 2007;67(19):9066-76

44. Hardy KM, et al. ErbB/EGF signaling and EMT in mammary development and breast cancer. J Mammary Gland Biol Neoplasia. 2010;15(2):191-9.

45. Wendt MK, Smith JA, Schiemann WP. Transforming growth factor$\beta$-induced epithelial-mesenchymal transition facilitates epidermal growth factor-dependent breast cancer progression. Oncogene. 2010;29(49):6485-98

46. Asiedu MK, et al. TGFbeta/TNF(alpha)-mediated epithelial-mesenchymal transition generates breast cancer stem cells with a claudin-low phenotype. Cancer Res. 2011;71(13):4707-19.

47. Xia LM, et al. Transcriptional up-regulation of FoxM1 in response to hypoxia is mediated by HIF-1. J Cell Biochem. 2009;106(2):247-56.

48. Pérez-Peña J, et al. Epigenetic modulation of FOXM1-gene interacting network by BET inhibitors in breast cancer. Breast Cancer Res Treat. 2018;172(3):725-32

49. Arceci A, et al. FOXM1 deubiquitination by USP21 regulates cell cycle progression and paclitaxel sensitivity in basal-like breast cancer. Cell Rep. 2019;26(11):3076-3086.e6.

50. Karunarathna U, et al. OTUB1 inhibits the ubiquitination and degradation of FOXM1 in breast cancer and epirubicin resistance. Oncogene. 2016;35(11):1433-44.

51. Ros $\mathrm{S}$, et al. Metabolic imaging detects resistance to pi3ka inhibition mediated by persistent FOXM1 expression in ER( + ) breast cancer. Cancer Cell. 2020;38(4):516-533.e9.

52. Li Q, et al. HIF-1a-induced expression of m6A reader YTHDF1 drives hypoxia-induced autophagy and malignancy of hepatocellular carcinoma by promoting ATG2A and ATG14 translation. Signal Transduct Target Ther. 2021;6(1):76.

53. Nishizawa Y, et al. Oncogene c-Myc promotes epitranscriptome $\mathrm{m}(6)$ A reader YTHDF1 expression in colorectal cancer. Oncotarget. 2018;9(7):7476-86.

54. Pi J, et al. YTHDF1 promotes gastric carcinogenesis by controlling translation of ZD7. Can Res. 2021;81(10):2651-65.

55. Han Z, et al. ALKBH5 regulates cardiomyocyte proliferation and heart regeneration by demethylating the mRNA of YTHDF1. Theranostics. 2021;11(6):3000-16

56. Xu C, et al. Prognostic values of YTHDF1 regulated negatively by mir-3436 in Glioma. J Cell Mol Med. 2020;24(13):7538-49.

\section{Publisher's Note}

Springer Nature remains neutral with regard to jurisdictional claims in published maps and institutional affiliations. 